\title{
Energy of Anticyclone
}

\author{
by \\ T. Ando \\ Meteorological Research Institute \\ (Received July 9, 1951)
}

\begin{abstract}
The various kinds of energies of anticyclones which are considered as the centers of action around Japan were calculated. The meaning of the potential energy and transformations of energies in the anticyclones were discussed.
\end{abstract}

\section{Calculation of energy}

In order to study energy balance in general circulation, the present author calculated energies of Siberia, Okhotsk and Ogasawara anticyc'ones which are considered to be the action center in the environment of Japan.

Though there are various kinds of energies, such as kinetic, potential, internal. or latent heat energies, the kinetic energy and the potential energy may be considered to be primary energies of the anticyclone, because water-vapour is not so important as a cyclone and a typhoon and also the internal energy is equal to $J c_{v} / R$ times of the potential energy.

The intensity of the anticyclone is defined by pressure difference at the center and its environment; then the potential energy of the anticyclone may also be defined by its comparative value to the environment. The value defined above is controlled largely by the temperature field and slightly by the pressure field. An anticyclone which is considered as an action center covers generally a large area and there are few upper air observations in it. Hence, we must often estimate its height and upper pressure only by surface observations.

The: height [1] of anticyclone $H$ is estimated by the following formula neglecting the deviation of lapse rate $\delta \beta$ from its environment.

$$
\text { (1. 1) } \quad H=-\begin{array}{ccc}
R & T_{0} \\
g & \frac{\delta p_{0}}{\delta T_{0}} & \frac{\delta}{\delta T_{0}}
\end{array}
$$

where $\mathrm{R}$ is gas constant, $g$ acceleration of gravity, $p_{0}$ surface pressure, $T_{0}$ surface temperature, and $\delta$ means the deviation from the environment. The calculated heights by (1.1) for some Siberia and Okhotsk anticyclones are in good agreement with observations as showh in Table 1.

Nex.t, the potential energy $U$ and its difference from the environment are given by the following formulae.

(1. 2)

$$
U=\int_{0}^{h} \rho g z d z=-p_{h} h+\bar{p} h
$$


Table $\mathbf{1}$

\begin{tabular}{c|c|c|c}
\hline & Cal. & Obs. & Diff. \\
\hline $\begin{array}{c}\text { Okhotsk } \\
\text { anticyclone }\end{array}$ & $5.6 \mathrm{~km}$ & $5.5 \mathrm{~km}$ & $0.1 \mathrm{ksa}$ \\
\cline { 2 - 4 } & 2.3 & 2.8 & 0.3 \\
\hline $\begin{array}{c}\text { Siberia } \\
\text { anticyclone }\end{array}$ & 4.3 & 4.0 & 0.3 \\
\cline { 2 - 4 } & 4.0 & 3.7 & 0.3 \\
\hline
\end{tabular}

(1. 3)

$$
\bar{p} h=\int_{0}^{h} p_{0}\left(\frac{T_{0}-\beta z}{T_{0}}\right)^{o / R \beta} d z
$$

where $\beta$ is lapse rate and assumed $\beta$ is constant from surface to height h. Transforming (1.2) by use of (1.3) and expanding it till the 5 th term, we have

(1. 4) $U=p_{0} h \times 10^{5}\left\{17.1 \frac{h}{T_{0}}-388.5 \frac{h^{2}}{T_{0}{ }^{2}}(1-0.03 \beta)+4974 \frac{h^{3}}{T_{0}{ }^{3}}(1-0.03 \beta)(1-0.06 \beta)\right\}$ where $z:$ in $\mathrm{km}, \beta:{ }^{\circ} \mathrm{C} / \mathrm{km}, g: 980 \mathrm{~cm} / \mathrm{sec}^{2}, \mathrm{R}: 2.8710^{6} \mathrm{erg} / \mathrm{gr} \mathrm{deg}$. Introducing (1.1) into (1.4) and differentiating, we have $\Delta \mathrm{U}$.

(1. 5) $\Delta \mathrm{U}=2.9 \times 10^{3} \Delta p_{0} T_{0} y\left\{y\left(\frac{1}{2}+\frac{1-0.03 \beta}{3} y+\frac{(1-0.03 \beta)(1-0.06 \beta)}{8} y^{2}\right)\right.$

$$
\left.-\left(\frac{1}{2}+\frac{2}{3}(1-0.03 \beta) y+\frac{3}{8}(1-0.03 \beta)(1-0.06 \beta) y^{2}\right)\right\}
$$

where $y=\frac{\Delta P_{0}}{p_{0}} \frac{T_{0}}{\Delta T_{0}^{\prime}}: \Delta$ means the difference from the environment. The area of an anticyclone is calculated by planimeter for every latitude. Kinetic energy $\mathrm{K}_{\mathrm{E}}$ is calculated from upper winds. When the data were not available, we calculated the gradient wind, because a wind in an anticyctone is considered to be a gradient wind. The results of calculations are showh in Tab!e 2 .

Table 2

\begin{tabular}{l|c|c|c}
\hline & $\begin{array}{c}\text { Siberia } \\
(1951, \text { Jan. 11) }\end{array}$ & $\begin{array}{c}\text { Okhotsk } \\
(1949, \text { Jun. 19) }\end{array}$ & $\begin{array}{c}\text { Oparawara } \\
(1950, \text { Lug. 9) }\end{array}$ \\
\hline$\Delta U$ & $2.0810^{27} \mathrm{erg}$ & $1.6810^{26} \mathrm{erg}$ & $4.6410^{26} \mathrm{erg}$ \\
$\mathrm{K}_{\mathrm{E}}$ & $3.0010^{25} \mathrm{erg}$ & $2.4810^{24} \mathrm{erg}$ & $5.1010^{24} \mathrm{erg}$ \\
\hline
\end{tabular}

We must notice in the above table that $\Delta \mathrm{U}$ is larger than $\mathrm{K}_{\mathrm{E}}$. As $\Delta \mathrm{U}$ is controlled by temperature field, $\Delta \mathrm{U}$ varies with the directions. For example, the cirtribution of sum of $\Delta U$ for unit cross-section for every $4 \mathrm{mb}$ in the case of Siberia anticyclone (1951, January 11) is larger for the south than for the north. Table 3 shows the distributions of $\Delta U$ above mentioned. It is interesting that the direction of outburst of cold air is the same with the direction of $\Delta \mathrm{U}$ maximum.

When we compare the above values with Kitty Typhoon (1948, August 30) for the unit volume, the latter on an average has 50-100 times of kinetic energy for the former. 
Table $\mathbf{3}$

\begin{tabular}{c|c|c|c|c|c}
\hline \multicolumn{1}{c|}{ Direction } & S & SE & NE & NW & W \\
\hline $\begin{array}{l}\text { Sum of potential } \\
\text { energy of unit } \\
\text { cross section }\end{array}$ & 126 & 120 & 106 & 73 & $8010^{5} \mathrm{erg}$ \\
\hline
\end{tabular}

\section{Meaning of $\Delta \mathrm{U}$}

Let $\rho$ be the average density in the anticyclonic cold dome and $\rho^{\prime}$ the average density in the environment, $H$ the height of the cold dome and which is a function of radius $r$, and $R$ the outerboundary of cold dome.

Then, by definition, $\Delta \mathrm{U}$ is written as follows

(2. 1)

$$
\begin{aligned}
\Delta \mathrm{U} & =\int_{0}^{R} \int_{0}^{H} \int_{0}^{2 \pi}\left(\rho-\rho^{\prime}\right) g z r d r d \theta d z \\
& =\int_{0}^{R} \int_{0}^{H} \int_{0}^{2 \pi} G z r d r d \theta d z \\
& =-\int_{0}^{R} \pi r^{2} G H \frac{\partial H}{\partial r} d r .
\end{aligned}
$$

where $G=\left(\rho-\rho^{\prime}\right) \mathrm{g}$. Wind $v_{\theta}$ in an anticyclone is considered in good approximation as a gradient wind.

Hence, $G \frac{\partial H}{\partial r}$ is given by gradient wind equation as follows.

$$
G^{\prime} \frac{\partial H}{\partial r}=g\left(\rho-\rho^{\prime}\right) \frac{\partial H}{\partial r}=\frac{\partial p}{\partial r}=\frac{\rho v_{\theta}^{2}}{r}+\rho \lambda_{\theta}
$$

where $\lambda$ is Coriolis' parameter. Introducing (2.2) into (2.1), we have

$$
\Delta \mathrm{U}=-K_{R}-\int_{0}^{R} \rho \lambda v_{\theta} \pi r^{2} H d r
$$

where $K_{E}$ is the kinetic energy of a tangential wind.

Next, we shall consider the resultant force which acts on the cold dome. Putting the northward force as $\mathrm{Y}$, and the eastward as $\mathrm{X}$, these are calculater as: follows.

(2. 4.)

$$
\begin{aligned}
X & =\int_{0}^{R} \int_{0}^{\pi} \int_{0}^{2 \pi} \rho \frac{d u}{d t} r d r d \theta d z \\
& =\int_{0}^{R} \int_{0}^{\pi} \int_{0}^{2 \pi} \rho \lambda v r d r d \theta d z-\int_{0}^{R} \int_{0}^{H} \int_{0}^{2 \pi} \frac{\partial p}{\partial y} \cdot d r d \theta d z \\
& =\int_{0}^{R} \int_{0}^{H} \int_{0}^{2 \pi} \rho\left(\lambda_{0}+\beta r \sin \theta\right)\left(v_{r} \sin \theta+v_{\theta} \cos \theta\right) r d r d \theta d z \\
& -\int_{0}^{R} \int_{0}^{\pi} \int_{0}^{2 \pi} \frac{\partial p}{\partial r} \cos \theta r d r d \theta d z \\
& =\rho \beta \int_{0}^{R} \pi r^{2} v_{r} H d r .
\end{aligned}
$$

And 
(2. 5)

$$
\begin{aligned}
Y & =\int_{0}^{R} \int_{0}^{H} \int_{0}^{2 \pi} \rho \frac{d v}{d t} r d r d \theta d z \\
& =\int_{0}^{R} \int_{0}^{H} \int_{0}^{2 \pi} \rho\left(\lambda_{0}+\beta r \sin \theta\right)\left(v_{\theta} \sin \theta-v_{r} \cos \theta\right) r d r d \theta d z \\
& -\int_{0}^{R} \int_{0}^{I} \int_{0}^{2 \pi} \frac{\partial p}{\partial r} \cdot \sin \theta d r d \theta d z \\
& =\rho \beta \int_{0}^{R} \pi r^{2} v_{0} H d r .
\end{aligned}
$$

where $\beta=\frac{\partial \lambda}{\partial y}$.

From (2.4) and (2.5), we have

(2. 6)

$$
X+i Y=\rho \beta \int_{0}^{R} \pi r^{2}\left(v_{r}+i \cdot v_{\theta}\right) H d r
$$

i) if $v_{\theta}>0, v_{r}<0$ as in a cyclone, then $Y>0, X<0$

ii) if $v_{\theta}<0, v_{r}>0$ as in an anticyclone, then $\mathrm{r}^{r}<0, \quad x>0$

Therefore, the resultant force acts southeastward for the anticyclone and northwest wardfor the cyclone, but this does not always mean that it moves in the specified direction. Combining (2.3) with (2.5), we have,

$$
K_{E}+\frac{\lambda}{\beta} Y=-\Delta U
$$

From $\S 1$, we know that $\Delta U \gg K_{E}$ for the anticyclone and so neglecting $K_{K}$, we have

$$
Y \approx-\frac{\beta}{\lambda} \Delta U
$$

Table 4

\begin{tabular}{|c|l|}
\hline Latitude & \multicolumn{1}{|c|}{$\beta / \lambda$} \\
\hline $20^{\circ}$ & $4.3410^{-8}$ \\
40 & 1.8710 \\
60 & 0.8910 \\
80 & 0.2810 \\
\hline
\end{tabular}

This expresses that the anticyclone is apt to move southward and the cycione northward because $\Delta U>0$ for the anticyclone and $\Delta U<0$ for the cyclone. Numerical values of $\beta / \lambda$ are showh in Table 4 . From the above Table, we see that $\mathrm{Y}$ is larger at lower latitudes for $\Delta U=$ const. and its value at lat. $20^{\circ}$ is about 15.5 times that of at lat. $80^{\circ}$.

Next, we shall consider the transformation of energy. Assuming pressure field as axially symmetrical, the equation of motion is written as follows:

then

$$
\frac{d v_{\theta}}{d t}+\frac{v_{r} v_{\theta}}{r}+\lambda v_{r}=0
$$

(2. 8$)$

$$
\frac{d \Omega}{d t}=0, \text { accrdingly, } \Omega=\text { const }=r\left(v_{\theta}+\frac{\lambda}{2} r\right)
$$

Let an air particle be at rest at $r=r_{0}$ near the center, and moves outwads spirally in the anticyclone, then by (2.8)

(2. 9$)$

$$
v_{0}=\frac{\lambda}{2}\left(\frac{r_{0}^{2}}{r}-r\right)=\frac{\lambda}{2}\left(\frac{r_{0}^{2}-r^{2}}{r}\right)=\frac{\lambda}{2 \pi}\left(\frac{s_{0}-s}{r}\right)
$$

where $s_{0}=\pi r_{0}^{2}, \quad s=\pi r^{2}$. 
Introducing (2.9) into (2.2), we have

$$
\frac{\partial H}{\partial r}=\frac{\lambda^{2}}{4 \pi G^{\prime}} \frac{s_{0}^{2}-s^{2}}{r \cdot s}
$$

where $G^{\prime}=\frac{G}{\rho}$. Integrating the above formula, we get

$$
H(r)=\frac{\lambda^{2}}{4 G^{\prime}}\left\{\left(\frac{R^{2}-r^{2}}{2}\right)-\frac{r_{0}{ }^{4}}{2}-\left(\frac{R^{2}-r^{2}}{R^{2} r^{2}}\right)\right\}
$$

As the first term on the right-hand side of above formula is larger than the second term, if we neglect the second term, we have,

$$
H\left(r^{\prime}\right)=\frac{\lambda^{2}}{8 G^{\prime}}\left(R^{2}-r^{2}\right)
$$

This is the same formula as derived by Rossby (1948) [2]. From (2.11), we know that $\mathrm{H}$ is large at high latitudes for fixed $\mathrm{R}$ and $\mathrm{r}$, for example, $\frac{H_{\lambda=60}}{H_{\lambda=30}}=1.73$.

Horizontal kinetic energy is written as follows:

$$
K_{E}=\frac{\rho}{2}\left(v_{\theta}^{2}+v_{r^{2}}{ }^{2}\right)=\frac{\rho}{8 \pi^{2} r^{2}}\left\{\lambda^{2}\left(s_{0}-s\right)^{2}+\left(\frac{d s}{d t}\right)^{2}\right\}
$$

Accordinly, when we use the equation of continuity, timely change of the kinetic energy are written as follows,

$$
\frac{d K_{w^{\prime}}}{d t}=\frac{\rho}{8 \pi} \frac{\partial w}{\partial z}\left\{\frac{\lambda^{2}\left(s_{0}{ }^{2}-s^{2}\right)-s^{2}\left(\frac{\partial w}{\partial z}\right)^{2}}{s}\right\}
$$

where $\lambda^{2} \gg\left(\frac{\partial w}{d z}\right)^{2}$, neglecting the second term, we have,

$$
\frac{d K_{T}}{d t}=\frac{\rho \lambda^{2}}{8 \pi} \frac{\partial w}{\partial z}\left(\frac{s_{0}^{2}-s^{2}}{s}\right)
$$

Integrating (2.12) for the total volume of the anticyclone and assuming,

we have

$$
w=w\left(1-\frac{r}{R_{*}}\right) \text {, }
$$

$$
\begin{aligned}
& \int_{0}^{R} \int_{0}^{I t} \int_{0}^{2 \pi} \frac{d K_{E}}{d t} r d r d \theta d z \\
& \quad=\frac{\pi \rho \lambda^{2} w}{4 R}\left\{r_{0}^{4} R \log \frac{R}{r_{0}}-\frac{3}{4} R r_{0}^{4}+\frac{4}{5} r_{0}{ }^{5}-\frac{R^{5}}{20}\right\}
\end{aligned}
$$

The value in the parentheses is in general negative and so (2.13) shows that if there are descending currents, kinetic energy will increase.

Next, we shall consider the potential energy of the anticyclone.

$$
\begin{aligned}
\int_{0}^{R} \int_{0}^{H} \int_{0}^{2 \pi} \frac{d \Delta U}{d t} r d r d \theta d z & =\int_{0}^{R} \int_{0}^{I t} \int_{0}^{2 \pi} G w r d r d \theta d z \\
& =\frac{\pi \rho \lambda^{2} w}{4}\left\{\frac{7}{60} R^{4}-r_{0}^{2}\left(\frac{R^{2}}{2}+\frac{r_{0}^{3}}{5 R}-\frac{r_{0}^{2}}{4}-\frac{r_{0} R}{3}\right)\right\}
\end{aligned}
$$

As the value in the parentheses of (2.14) is in general positive, a descending mo- 
tion acts as an influence to decrease potential energy and to increase kinetic energy. When we consider the magnitude of each term in (2.13) and (2.14), $-\frac{1}{20} R^{5}$ and $\frac{7}{60} R^{4}$ are the greatest among them and so we have approximate forulae as follows:

$$
\begin{aligned}
& \int_{0}^{R} \int_{0}^{I I} \int_{0}^{2 \tau} \frac{d K_{F}}{d t} r d r d \theta d z=-\frac{\pi \rho \lambda^{2} w}{80} R^{4} \\
& \int_{0}^{R} \int_{0}^{I I} \int_{0}^{2 \pi} \frac{d \Delta U}{d t} \cdot d r d \theta d z=\frac{\pi \rho \lambda^{2} w}{80} 2.3 R^{4}
\end{aligned}
$$

Adding (2.15) and (2.16), we have $\frac{d Y}{d t}$ from (2.7)

$$
\frac{d Y}{d t}=\frac{\beta}{\lambda} \int_{0}^{R} \int_{0}^{I t} \int_{0}^{2 \pi} \frac{d\left(K_{K}+\Delta U\right)}{d t} r d r d \theta d z=-\frac{\pi \rho \lambda \beta w}{80} 1.3 R^{4}
$$

If $w<0$, as in an anticyclone, $\frac{d Y}{d t}>0$, then its southward. resultant force will decrease. Magnitude of $\beta \lambda$ is maximum in midde latitudes shown in Table 5 .

Table 5

\begin{tabular}{c|rrrr}
\hline Latitude & $80^{\circ}$ & $60^{\circ}$ & $40^{\circ}$ & $20^{\circ}$ \\
\hline$\beta \lambda$ & 5.75 & 14.44 & 16.20 & $10.7010^{-19}$ \\
\hline
\end{tabular}

From this table, we can suppose that $(2,17)$ would be one reason that an anicyclone moving southward recurves toward the north in middle latitudes.

From the relations between $(2.15),(2.16)$, we can find the energy transformation. That is to say, potential energy will decrease by descending motion and about $43 \%$ of its decrement convert into kinetic energy.

Acknowledgment - The present author is deeply indebted to Dr.K. TAKAHASHI his guidance and encouragement through this work.

\section{References}

[1] Hoshino, T. 1947: On the Height of an Anticyclone, Journal of Meteoeological Society of Japan, 25, p. 10.

[2] Rosssy, C.G. 1948: On Displacement and Intensity Changes of Atınospheric Vortices, Journal of Marine Research, 5, p. 175. 